\title{
Travels to High Altitudes with Cardiovascular Diseases
}

\author{
Emrush Rexhaj and Rodrigo Soria Maldonado \\ Departments of Cardiology and Biomedical Research, University of Bern, Switzerland
}

Travelling to high altitudes has become very popular over the last years, not only for seasoned alpinists but also for tourists in general. Thus, an increasing number of tourist agencies propose high-altitude experience to potential customers. However, extreme environments $(>2500 \mathrm{~m}$ above sea level) are characterized by hypoxemia and require a series of respiratory and cardiovascular adjustments to maintain proper oxygen delivery to the different organ systems. These adjustments occur via chemoreflexes involving the sympathico-adrenal system [1].

Unfortunately, not everyone reacts in the same way to high-altitude exposure, and even healthy people may suffer from high altitude-related diseases, i.e., acute mountain sickness (AMS), high-altitude pulmonary oedema (HAPE), and high-altitude cerebral oedema (HACE) [2]. In patients with pre-existing cardiovascular diseases, these adaptive mechanisms take place already at sea level, and hypoxic environments are risky for these persons [3]. While travelling to moderate altitudes $(<2500 \mathrm{~m})$ is considered safe for patients with stable cardiovascular diseases, careful and individualized evaluation is wanted in patients with instable conditions prior to exposure to higher altitudes $>2500 \mathrm{~m}$.

Jan Vontobel, in his review "Herzpatienten und Höhenexposition" [4] in this issue of "Praxis", demonstrates the challenges at hand in a comprehensive manner and supplies practical recommendations that need to be considered when advising patients before travelling to high-altitude locations. The available evidence from published studies summarized by Vontobel seems not to be too strong, since most of the evidence has a grade of recommendation between B and C. Nevertheless, the level of class evidence is quite high, between 1 to lla. In each patient, general recommendations such as acclimatization, avoiding overexertion, avoiding nonstop access to an altitude $>2500 \mathrm{~m}$, maintaining hydration and adapting treatments should be part of the initial patient evaluation and counseling. Downright contraindications as proposed in Table 1 must not be ignored.

In all and due to the high individual variability of the adaptive response to high altitude exposure and the moderate strength of evidence, it is preferable to follow the line of a cautious approach with patients with underlying cardiovascular diseases. Careful pre-exposure assessment, especially taking into account the patient's disease and history, may minimize risks and highlight contraindications to high altitude exposure.
Table 1. Contraindications to high altitude exposure (modified from [5])

\begin{tabular}{ll} 
Cardiovascular disease & Contraindication when: \\
\hline Arterial Hypertension & $\geq 160 / 100 \mathrm{mmHg}$ at rest \\
& Systolic blood pressure $>220 \mathrm{mmHg}$ during \\
& excercise
\end{tabular}

\section{References}

1. Rimoldi SF, Sartori C, Seiler C, et al. High-altitude exposure in patients with cardiovascular disease: risk assessment and practical recommendations. Prog Cardiovasc Dis. 2010;52(6): 512-524.

2. Bärtsch P, Swenson ER. Clinical practice: Acute high-altitude illnesses. N Engl J Med. 2013;368(24):2294-2302.

3. Parati G, Agostoni P, Basnyat B, et al. Clinical recommendations for high altitude exposure of individuals with pre-existing cardiovascular conditions: A joint statement by the European Society of Cardiology, the Council on Hypertension of the European Society of Cardiology, the European Society of Hypertension, the International Society of Mountain Medicine, the Italian Society of Hypertension and the Italian Society of Mountain Medicine. Eur Heart J. 2018;39(17):1546-1554.

4. Vontobel J: Herzpatienten und Höhenexposition. Praxis. 2021;110:303-311. 
5. Hofstetter L, Scherrer U, Rimoldi S. Going to high altitude with heart disease. Cardiovasc Med. 2017;20(04):87-95.

\section{Emrush Rexhaj, MD}

Department of Cardiology

Inselspital, University Hospital Bern

3010 Bern

Switzerland

emrush.rexhaj@insel.ch
German version online at: https://econtent.hogrefe.com/ doi/abs/10.1024/1661-8157/a003653

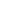

